\title{
Sublinguale Therapie hilft auch perennial
}

Eine Wirksamkeit der sublingualen Immuntherapie bei der saisonalen allergischen Rhinitis des Erwachsenen ist nachgewiesen. Für die perenniale Rhinitis war die Datenlage bisher unzureichend. Eine kleine Doppelblindstudie kann nun bei dieser Indikation Erfolge vorweisen.

_ ranzösische Allergologen versorgten 120 Patienten mit einer perennialen Rhinitis - manche auch mit moderatem Asthma - aufgrund einer Hausstaubmilbensensibilisierung mit milbendichten Matratzenüberzügen und selektierten nach 6 Wochen die Patienten, die immer noch unter erheblichen nasalen Beschwerden litten (Score von über 70 bei 168 möglichen Punkten). Von ihnen erhielten randomisiert und doppelblind 15 Patienten 2 Jahre lang sublingual Allergene von Dermatophagoides pteronyssinus and $D$. farinae im Verhältnis $1: 1,17$ Patienten erhielten Plazebo. In der Auf- titrationsphase wurden Tropfen, in der Erhaltungsphase Tabletten gegeben.

Fünf Patienten in der Verum- und neun Patienten in der Plazebogruppe verließen die Studie vorzeitig. Im Gesamtscore war bereits nach 6 Monaten ein deutlicher Unterschied zwischen beiden Gruppen feststellbar. Nach dem ersten und zweiten Jahr war die Immuntherapie der Plazebogabe dann signifikant überlegen ( $\mathrm{p}<0,03$ bzw. $\mathrm{p}<0,02$ ). Auch in der Aufschlüsselung nach Einzelsymptomen zeigte sich ein Unterschied zugunsten von Verum bei den Symptomen nasales Jucken und verstopfte Nase (jeweils $\mathrm{p}<0,01)$. Der quantitative Hautpricktest mit beiden Milbenarten ergab eine signifikante Abnahme der Reaktivität in der Verumgruppe $(p<0,03)$. Beim Medikationsscore ließ sich kein signifikanter Unterschied errechnen, allerdings war der Medikamentenverbrauch in beiden Gruppen sehr niedrig. Schwere oder systemische Nebenwirkungen der Behandlung traten nicht auf.

Fazit: Sowohl bei den klinischen Symptomen (Rhinitisscore) als auch bei der Reaktivität auf die Allergene im Hauttest ergab sich in dieser Studie bei Patienten mit einer perennialen Rhinitis eine Überlegenheit der SLIT gegenüber Plazebo. Die Applikation als Tablette könnte die Patientenakzeptanz erhöhen.

no

Tonnel AB et al. Allergic rhinitis due to house dust mites: evaluation of the efficacy of specific sublingual immunotherapy. Allergy 2004; 59: 491-7

\section{Tags schläfrig, nachts schlaflos?}

\section{Patienten mit Heuschnupfen schlafen schlecht und sind tagsüber oft müde - so die gängige Meinung. Mannheimer HNO-Ärzte unter- nahmen jetzt einen Versuch, diese Annahme mit objektiven Daten zu unterfüttern.}

m Rahmen einer prospektiven kontrollierten klinischen Studie wurden 25 Patienten mit saisonaler allergischer Rhinitis und 25 gesunde Probanden untersucht. Alle Teilnehmer mussten sich sowohl vor als auch während der Pollensaison je 2 Nächte im Schlaflabor einer Polysomnographie unterziehen. Die klinische Symptomatik wurde durch einen Rhinitiscore, die Schläfrigkeit am Tag mit der „Epworth Sleepiness Scale“ (ESS) erfasst.

Während der Pollensaison betrug der Rhinitissymptomscore der Allergiker 21,3 gegenüber 1,05 in der pollenfreien Zeit. Neun Patienten hatten eine leichte, sieben eine mäßige und acht eine schwere Rhinitis. Kein Proband der Kontrollgruppe zeigte allergische Symptome.

Die mit der ESS erfasste Müdigkeit am Tag lag außerhalb der Pollensaison in beiden Gruppen im Bereich des Nor- malen. Während der Pollensaison steigerte sich der ESS-Wert bei den Allergikern im Mittel von 6,8 auf 8,2, in der Kontrollgruppe blieb er unverändert ( $\mathrm{p}=0,048)$. Die individuelle Zunahme der Tagesmüdigkeit bei den Allergikern korrelierte mit dem Symptomscore.

Bei den in der Polysomnographie erhobenen Daten ergaben sich signifikante Unterschiede bei den Parametern Schnarchen und Sauerstoffsättigung, insgesamt bewegte sich jedoch die Effektivität des Nachtschlafes in allen Gruppen während und außerhalb der Saison - im Normbereich. Die Tagesmüdigkeit der Rhinitiker ist also nicht schlüssig durch fehlenden Nachtschlaf zu erklären.

Fazit: Während der Pollensaison leiden Patienten mit allergischer Rhinitis unter einer erhöhten Tagesmüdigkeit. Diese ist weniger auf Schlafmangel während

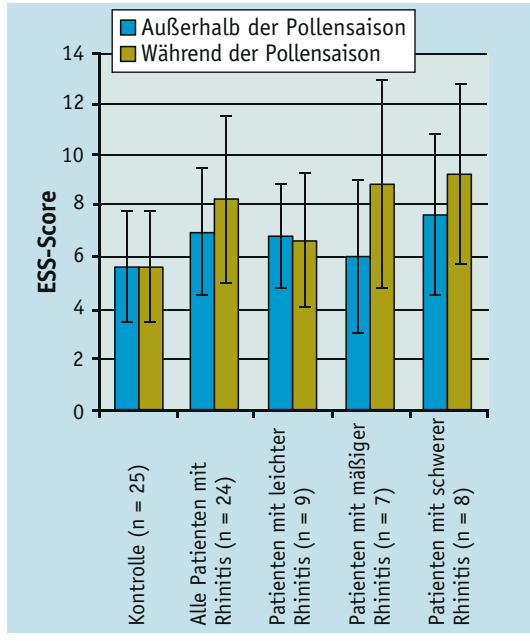

Müdigkeit tagsüber, gemessen mit der „Epworth Sleepiness Scale“ (ESS), von Rhinitispatienten während und außerhalb der Pollensaison

der Nacht zurückzuführen, sondern scheint vielmehr durch die Krankheit selbst verursacht zu sein

Stuck BA et al. Changes in daytime sleepiness, quality of life, and ojective sleep patterns in seasonal allergic rhinitis: a controlled clinical trial. J Allergy Clin Immunol 2004; 113: 663-8 